\title{
Le brouillage des identités: nouvel enjeu de la francophonie
}

\author{
Laura LóPEZ MORALES \\ Universidad Nacional Autónoma de México
}

\begin{abstract}
Plus que jamais la migration est un phénomène global. Plus de cent millions de personnes vivent aujourd'hui hors de leur pays natal. Ce n'est plus le Cahier d'un retour au pays natal qu'il faudrait lire, mais celui de la fuite du pays natal.
\end{abstract}

\section{Joël Des Rosiers}

Le paysage identitaire de ce tournant non seulement du siècle mais du millénaire est défini par l'hétérogène. La carte démographique que nous pouvons tracer surtout dans les pays industrialisés met en évidence la diversité croissante des groupes qui composent leur population. Or ce phénomène, résultat de différents processus d'ordre social, politique, économique et culturel, s'est considérablement accentué au cours des dernières décennies et a entraîné une série de conséquences de la plus grande importance.

La Francophonie comme espace géographique éclaté n'échappe pas à cette problématique et, dans la mesure où les anciens repères de "territoire, langue, culture, origine", ne permettent plus tout à fait de définir une identité, il convient de les réviser et, surtout, d'y introduire de nouvelles données imposées par les conditions actuelles. Ceci représente un défi de taille qui ne sera relevé que grâce à une conscience bien claire des acteurs et des facteurs en présence; c'est seulement à cette condition qu'on mettra à profit l'inépuisable richesse que peut représenter la pluralité, la diversité, la différence, autrement dit, la possibilité du métissage culturel dans la tolérance et le respect.

La problématique de l'identité remet donc inéluctablement sur le tapis des notions on ne peut plus polémiques, comme celles que nous venons d'évoquer et sur lesquelles nous ferons uniquement quelques remarques globales nécessaires à notre exposé. En effet, les spécialistes eux-mêmes n'ont pas épuisé la discussion à propos de ce que l'on entend par culture, origine, 
race, territoire, langue... comme critères identitaires. Et pour renchérir, les notions en question en appellent d'autres également importantes: métissage, altérité, hétérogénéité, diversité, pluralité... qui constituent le réseau thématique de nos propos.

Les quelques commentaires autour de ces termes clé auront pour fonction de baliser le terrain qui nous intéresse plus particulièrement, à savoir l'espace littéraire dans lequel nous trouvons doublement incarnée cette dimension du paysage actuel de la francophonie: la présence de plus en plus visible d'écrivains' d'origines multiples et diverses établis en terres francophones et la manière dont, à travers leurs écrits, ils vivent et expriment cette expérience de déracinement/enracinement ${ }^{2}$ nous offre la possibilité de nous interroger sur la façon dont se construisent les nouveaux profils identitaires des générations actuelles et, peut-être, de celles à venir. Les témoignages de ces êtres déchirés entre un ailleurs originaire et un ici le plus souvent hostile et qui se demandent d'une manière obsédante "Qui sommes-nous?", ne peuvent pas être ignorés de leurs voisins autochtones. Or le moins qu'on puisse dire c'est que la littérature francophone contemporaine renouvelle l'imaginaire des uns et des autres car elle permet de "mesurer l'écart qui [les] sépare de [leur] pays rêvé et de [leur] pays réel". ${ }^{3}$ C'est entre ces deux "pôles" que s'attarde, par exemple, le dernier livre publié par Dany Laferrière (1999), écrivain haïtien, émigré au Québec depuis plus d'une vingtaine d'années.

\section{Quelques remarques préalables}

Dès que nous parlons d'identité nous sommes automatiquement confrontés à la polémique de la notion de culture et, par ricochet, à celle de langue. Dans son article "Langues, cultures et territoires, quels rapports?" (1995), Jean Lafontant, universitaire d'origine haïtienne résidant au Canada depuis plus de quinze ans, s'interroge sur le bien fondé des rapports automatiques que l'on établit entre ces notions et il apporte des arguments de poids pour réviser ce trinôme à la lumière de ce qui se passe de nos jours dans les

1 Mais également d'artistes, d'intellectuels et autres citoyens anonymes.

${ }^{2}$ Le cas de la littérature "beure" n'en est qu'une variante dans une importante production signée par des immigrants d'origines différentes installés depuis une ou deux générations en France. Et on pourrait en dire autant à propos d'autres pays européens.

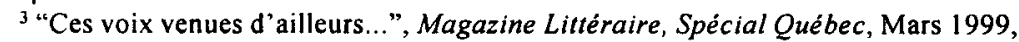
p. 196. 
régions à fort taux d'immigration. Nous retiendrons de son argumentation ce qu'il avance à propos de la notion de culture.

"Sa définition la plus courante présente [la culture] comme un héritage traditionnel de valeurs, normes, symboles et rituels particuliers transmis surtout par le processus de socialisation" $(1995,230)$. Rappelons aussitôt que notre intérêt pour aborder la question, en fonction de ce qui se passe actuellement dans les contextes pluriethniques, nous oblige d accepter le suite de l'argumentation de Lafontant: "une culture ne fait pas que se transmettre. Elle (c'est-à-dire les sujets qui la portent) affronte d'autres cultures, d'où des processus d'emprunts, d'échanges, de réinterprétations..." qui correspondent à ce que, dans les années 40 , l'anthropologue cubain Fernando Ortiz a désigné sous le nom de transculturation.

Le risque est donc grand de vouloir faire prévaloir les critères de moeurs, traditions, ethnie, peuple, etc... dans la définition de culture car on est vite sur le territoire des revendications nationalistes, xénophones et autres intégrismes. Pour sa part, Jean-Loup Amselle, anthropologue lui aussi, nous met en garde contre un tel danger: "Dans tout culturalisme, comme dans tout nationalisme d'ailleurs, il y a un fondamentalisme qui sommeille" $(1990,62)$.

Dans un essai, au titre on ne peut plus révélateur et provocateur: Les Identités meurtrières (1998), l'écrivain franco-libanais Amin Maalouf, reprenant son cas personnel, déclare que lorsqu'on le presse de décliner s'il se sent Libanais ou Français, ou moitié-moitié, se sent obligé d'affirmer que "en tout homme se rencontrent des appartenances multiples..."; les immigrés du monde entier sont "des êtres transfrontaliers [...] traversés par des lignes de fracture ethniques, religieuses ou autres" (p. 13). Tout au long de son texte, l'auteur de Léon l'A fricain s'efforce d'alerter le lecteur contre les risques et les méfaits qu'entraîne la volonté de classer les individus sous des étiquettes qui les compartimentent et souvent les opposent de manière meutrière. Ce qu'il veut c'est "comprendre pourquoi tant de personnes commettent aujourd'hui des crimes au nom de leur identité religieuse, ethnique, nationale ou autre" (p. 17).

Dans le contexte québécois actuel, comme c'est le cas aussi dans d'autres espaces européens, "le foisonnement de signes identitaires surcharge les paysages culturels, et les possibilités pour l'individu de se reconnaître dans un ensemble s'en trouvent brouillées. [...] ce sont les catégories mêmes du proche et du lointain, du familier et de l'étranger, du semblable et du différent qui se trouvent confondues", nous précise Pierre Nepveu (1999, pp. 199200). Plus loin nous verrons à l'oeuvre quelques exemples littéraires de ce brouillage pour montrer que la perception de la culture comme une totalité ne peut qu'être remise en question. 
Dans ces conditions, il n'y a plus ni totalité ni continuité de l'ensemble de moeurs et de traditions qui puisse constituer la spécificité d'un groupe dont tel ou tel individu se réclame et les rapports d'altérité cessent d'être vécus comme une pure différence exotique. Les référents du nous et des autres deviennent mouvants de sorte que, souvent, les données actuelles rendent peu opératoires les notions de "communauté", d'un nous renvoyant à une réalité cernable. Les différents nous deviennent fragmentaires et parfois désagrégés. En fait, les sociétés et la culture occidentales loin d'incarner une supposée homogénéité, se définissent davantage aujourd'hui, comme nous l'avons signalé, par l'hétérogéneité.

En effet, l'expérience de l'étrangeté est de plus en plus fréquente dans les sociétés avec un pourcentage croissant d'immigrants qui ne sont plus attirés automatiquement par la culture d'accueil, au départ promesse de paradis. Vivant comme une contrainte l'appropriation des modèles de la société d'adoption, ils ne sont plus tellement disposés à renoncer à leurs particularités. Sergio Kokis, romancier d'origine brésilienne, installé au Québec depuis une vingtaine d'années, fait dire au narrateur de son premier roman:4 "Malgré ma compréhension du francais, je me rendais sans cesse compte de mon incapacité à dire les mêmes choses que les autres. Les visages dans le métro, l'odeur des gens, leur quotidien, leurs gestes, grimaces et stéréotypies de langage rendaient mon dépaysement chaque fois plus profond" (p. 201).

Dans le contexte francais, cette étrangeté est formulée comme suit par Leîla Sebbar (1986), romancière algérienne vivant en France depuis les années 70 :

Je n'ai pas, après tant d'années, réussi à acquérir la souplesse, l'intelligence qui me permettraient la pratique efficace d'un certain nombre de codes sociaux, culturels, mondains que je connais et qui me précipitent chaque fois dans un mutisme obstiné et stupide. Souvent, j'ai été frappée chez toi [Nancy Houston ] par cette capacité que tu as, je l'ai remarquée chez d'autres femmes en exil, [...] d'assimiler et d'utiliser les codes les plus complexes, sans s'y conformer totalement, sans servilité. Peut-être la différence tient au fait que ces femmes, dont tu fais partie à mes yeux, ont été policées par des siècles de culture,

4 Le Pavillon des miroirs, 1995, lauréat de quatre des plus importants prix littéraraires du Québec.

${ }^{5}$ Sebbar a entretenu pendant plus de deux ans une correspondance avec la romancière canadienne Nancy Houston dans laquelle elles traitent de leur travail littéraire, mais surtout de leur expérience d'exilées. 
culture d'origine européenne, j'entends, la culture dominante, alors que les réfractaires dont je serais en partie - avec d'autres femmes du $<<$ tiers monde $>>$, pays en voie de développement, pays en sous - et en maldéveloppement -auraient été maladivement contaminées par les effets de la colonisation (pp. 9-10).

\section{Ni totalité ni continuité}

Reprenons à ce point une autre des notions évoquées au commencement de cet exposé: le territoire, autrement dit, la dimension spatiale dont il faut tenir compte dès qu'il s'agit d'expliquer la dynamique, sur un espace donné, entre individus/groupes qui y sont installés depuis longtemps et ceux aux origines géographiques diverses et, souvent, très récemment arrivés dans le pays d'adoption. ${ }^{6}$ Or, c'est justement la notion d'espace qui est venue modifier la réflexion, car la remise en question de la culture comme une totalité et une continuité de traditions déplace la perspective historique au bénéfice d'une approche plutôt horizontale et synchronique. Autrement dit, l'objet d'analyse est le comportement et la dynamique des différents groupes sociaux dans l'espace d'accueil, notamment l'espace québécois.

Il en résulte que le champ de la culture renvoie à une prolifération de signes, à une diversité accrue de représentations donnant lieu à autant d'interprétations que de groupes se disputant, avec des discours différents, sinon la dominance sur les autres du moins la reconnaisance de leur différence et de leur volonté ou non d'intégration. C'est pourquoi à l'intérieur de cet espace culturel les frontières qui séparent les groupes, en raison de leur caractère mouvant, sont source de nouveaux sens qu'il s'agit d'apprendre à identifier et à décoder. "Tout noter. Ne rien oublier. L'urgence..." revient comme un martèlement dans La Québécoite de Régine Robin (1983). Pour la narratrice il s'agit de "tout emmagasiner, comme si tu devais te retrouver tel Robinson sur son île et ne plus rencontrer Montréal que par ces traces, signes, symboles, fragments sans signification, morceaux, débris, tessons hors d'usage" (p. 203).

La réalité objective, si tant est qu'elle existe, n'est pas percue ou interprétée de la même manière d'un côté ou de l'autre de telle ou telle frontière tracée dans un même espace culturel. Les sens qui en émergent ne peuvent être que différents selon que les groupes qui les créent et les

'On pourrait ajouter à ce tissu référentiel le territoire d'origine des immigrants, "les ailleurs" auxquels ils renvoient souvent dans leur nostalgie d'exilés. 
déchiffrent se trouvent au centre ou sur les marges. Alors, du moment où la notion de culture cesse d'être le fondement unitaire des groupes en présence, la revendication identitaire se trouve "brouillée" par rapport aux anciennes affiliations collectives.

\section{De l'approche verticale à l'approche horizontale}

Le cas du Québec est exemplaire en ce qui concerne le passage de l'approche historique ou verticale à une perspective horizontale ou synchronique de la culture. En effet, jusqu'aux années 70 et début des années 80 , dans cette province, qui tout au long de son histoire avait brandi le drapeau de l'identité pour se donner une justification surtout à ses propres yeux, la perception de sa culture était associée à l'idée de "pays colonisé", puisque le "rapport colonial" expliquait ainsi l'aliénation culturelle et l'expérience de la dépossession. Il faut néanmoins préciser que cette lecture historique avait oublié ou sous-estimé une dimension capitale dans la compréhension du fait québécois contemporain, que les approches sociologiques récentes oni récupérée et analysée dans toute sa portée: le profil culturel québécois pris sous cet angle ne mettait pas en scène tous les acteurs sociaux et culturels en présence. Le changement vers une approche plutôt spatiale a mis en évidence, au cours de ces décennies, qu'il existait au moins deux autres forces agissant sur l'évolution et le paysage sociodémographique, et donc culturel, du Québec: les Amérindiens et les immigrants. ${ }^{7}$ On s'est alors apercu que la présence et le poids incontournables de ces acteurs rendaient plus complexes et plus conflictuelles les perceptions de l'identité et de la différence. Au terme de ce processus la littérature québécoise a pris conscience qu'elle était passée, presque sans s'en rendre compte, de la phase d'une quête obsédante de l'identité à celle où l'imaginaire était plutôt habité par le désir et la liberté, mais avait aussi incorporé d'autres points de référence.

Le chercheur français Jean-Michel Lacroix, cité par Benoît Melançon (1990), affirme que "le Québec a [longtemps] pris tout l'espace et ne s'est pas montré sensible aux écritures élaborées dans le même territoire et produites par des langues et des cultures différentes". C'est grâce à d'autres chercheurs, canadiens cette fois-ci, qu'on a comblé cette carence. ${ }^{8} \mathrm{Et}$

7 fl faut tout de même signaler que Yves Thériault a été l'un des rares écrivains à leur avoir accordé, depuis les années cinquante, une place importante dans ses récits. On y trouve autant le monde amérindien que des personnages issus de l'immigration.

" Notamment Sherry Simon, Pierre Nepveu et Simon Harel. 
Melançon lui-même de souligner, en reprenant Pierre Nepveu, que le "fait important qui caractérise l'écriture migrante des années 80 , c'est sa coïncidence avec tout un mouvement pour lequel, justement, le métissage, l'hybridation, le pluriel, le déracinement sont des modes privilégiés, comme, sur le plan formel, le retour du narratif, des références autobiographiques, de la représentation (p. 201).

\section{Les identités révisitées}

Au cours de la même période, le discours critique contre le nationalisme, l'écriture féminine et la parole immigrante, installent à nouveau sur le tapis la question de l'identité, seulement, cette fois-ci, dans la perspective de l'hétérogène.

La Québécoite (1983) de Régine Robin est on ne peut plus représentatif de ce besoin et de cette volonté de défaire, depuis la position de l'étranger, de l'immigrant, le cercle "protecteur" du nous québécois.

Par moments, cependant, ces moments qui reviendraient souvent où son mari lui ferait sentir qu'elle n'était pas d'ici, elle hésiterait. La peur. [...] La peur de l'homogénéité

de l'unanimité

du Nous excluant tous les autres

du pure laine

elle l'immigrante

la différente

la déviante

Elle hésiterait

Car il pourrait aussi y avoir une façon québécoise de faire

la chasse aux sorcières

car il pourrait aussi y avoir une façon québécoise

d'être xénophobe et

antisémite (p. 133).

On est donc face à une nouvelle étape ouverte à l'émergence d'autres expressions identitaires marquées par l'altérité et qui mettent en échec le discours officiel réflétant une homogénéité supposée. En effet, pendant longtemps et plus nettement depuis la Révolution Tranquille, les voix s'étaient accordées pour clamer et défendre la construction d'une littérature nationale qui exprimerait les assises et les valeurs authentiques du peuple québécois. Dans ce nous, prétendument créateur, acteur et destinataire de ce discours, 
il n'y avait pas de place pour l'hétérogène, pour ce qui était de l'autre côté des frontières imaginaires tracées par cette première personne du pluriel. Jusqu'alors l'altérité "étrangère" était essentiellement incarnée par les anglocanadiens. Mais Régine Robin ne fait autre chose que souligner la distance dont elle se sent séparée par rapport au nous québécois.

Cette parole immigrante que l'on ne peut ni accrocher, ni fixer, ni qualifier, assume, en faisant migrer les images, le rôle de convoyeuse de l'hétérogène. Dans le cas concret de cette romancière, comme ceux aussi de Sebbar et de Kokis, et de tant d'autres, l'impossibilité de se rattacher à un noyau territoire/ identité originaire renforce le sentiment d'exil et de déterritorialisation. Pour elle, le nouveau "cosmopolitisme" consiste à traverser les codes, à en jouer, à développer une parole nomade qui ne soit pas parole d'exil, à travailler cette bordure où parole migrante et parole immigrante se conjuguent, à tenir à "ce défaut d'identité et à cette identité molle..." (cité par L'Hérault, 1991, 69). Dans cette errance identitaire, elle ne reconnaît d'autre territoire que la langue, la parole. Obsédée qu'elle est par le besoin d'accrocher à son nouveau contexte, elle veut tout retenir: "Oui, noter toutes les différences. Ne rien oublier, ni les marques de dentifrices, les chaînes de Barbecue, celles de Pizzas, ni les marques de savon ou de lessive. Pénétrer l'étrangeté de ce quotidien. En exil dans ta propre langue. Le leurre de la langue. Ni la même, ni une autre. L'AUTRE dans le MÊME" (p. 183).

Encore une fois, point n'est besoin de rappeler combien, tout au long des dernières décennies, la notion d'identité s'est chargée de différentes connotations idéologiques selon les perspectives d'approche. Pour sa part, le discours féministe, comme nous l'avons signalé, n'a pas manqué d'introduire sa propre lecture du binome identité/altérité sans s'arrêter à la distinction traditionnelle femme/homme, mais allant à un autres plus vaste et diversifié. C'est ce que France Théoret propose lorsqu'elle affirme qu'elle veut l'identité féminine "ouverte et multiple dans l'accès aux cultures" (cité par L'Hérault, 1991, 70).

\section{Les lettres: miroir de l'hétérogène}

Nous venons donc de voir comment, dans les années 80 , la production romanesque au Québec aborde de plus en plus ouvertement le thème de l'immigration, de l'expérience coloniale ou de l'altérité culturelle. Occupant le devant de la scène ou inscrits dans le paysage social qui sert de cadre à l'histoire, l'autre, l'étranger, le migrant, défilent de plus en plus dans l'espace littéraire québécois. 
Chez Francine Noël ces représentants de l'hétérogène deviennent parfois source d'inquiétude ou de malaise pour Fatima dans Nous avons tous découvert l'Amérique (1990). Mais ces "autres" peuvent également incarner la possibilité de combler ses propres carences identitaires; pour la narratrice de ce roman, le lien affectif le plus fort est celui qu'elle entretient avec Amélia, l'amie latinoaméricaine, l'âme jumelle trop tôt arrachée par la mort et qui la laisse mutilée d'une partie essentielle de son être-au-monde.

Sans constituer sa préoccupation centrale, Juliette Pomerleau, la généreuse et monumentale protagoniste du roman éponyme de Yves Beauchemin, compte parmi ses locataires un musicien tchèque. Pour traiter son obésité, on conseille à Juliette d'aller voir le Dr. Yong, spécialiste chinois en acupuncture. Enfin, Vinh est un petit vietnamien ami de Denis, le neveu de Juliette. La présence des étrangers est de plus en plus fréquente et naturelle dans le paysage social canadien en général. Et ainsi on pourrait citer d'autres écrivains québécois dont l'oeuvre capte cette dimension de leur réalité.

A leur tour, les personnages créés par les écrivains venus d'ailleurs apparaissent comme emblématiques d'un groupe culturel, ou mettent en relief les effets que le contact entre cultures différentes produisent chez les individus appartenant autant à la communauté "de souche" qu'aux dites "communautés ethniques". Nous trouvons dans L'Ecologie du réel (Nepveu, 1999) un échantillonage assez représentatif des écrivains de cette période dans le chapitre consacré aux "Ecritures migrantes".

Par ailleurs, cédant à la mode des théories culturalistes bâties sur la notion d'ethnicité et qui ont eu alors leur moment de gloire, ces oeuvres ont été perçues non pas comme un phénomène textuel d'interférence, mais plutôt comme des produits définis par l'origine des auteurs, par les problèmes d'intégration ou de mobilité sociale. D'autres approches visaient plutôt à réduire l'ethnicité à la confrontation de codes liés à la mémoire, aux mémoires multiples et fragmentaires. En fait, l'ethnicité ainsi vécue équivalait plutôt à une marginalité culturelle.

Il convient à ce sujet de rappeler que l'option politique du multiculturalisme - tel que pratiquée par le Canada- doit être envisagée avec énormément de prudence car les risques de dérapage sont nombreux. Aussi, Neil Bissoondath, l'écrivain canadien né à Trinidad mais dont les parents venaient de l'Inde, expose-t-il ses réserves dans un livre qui a fait couler beaucoup d'encre au moment de sa publication. Le marché aux illusions. La méprise du multiculturalisme (1995): "Le multiculturalisme [...] n'a rien fait --et ne peut rien faire-- pour nous aider à construire une idée réaliste et lucide de nos voisins. Reposant sur des stéréotypes garantissant que les groupes ethniques vont préserver leur caractère distinct dans une forme douce 
et insidieuse d'appartheid, le multiculturalisme n'a pas réussi à faire plus qu'engager un pays déjà divisé sur le chemin d'une plus grande division sociale" (p. 102). Bissoondath n'est pas le seul à mettre en garde ceux qui croient que cette formule est la voie à suivre par les sociétés pluriethniques; il rapporte les propos du recteur de l'UQAM, Michel Corbo, pour qui cette politique "a empêché plusieurs $<<$ de s'intégrer naturellement au tissu de la société canadienne et québécoise >" (p. 209).

Discours romanesque et exil

Le roman n'est pas une confession de l'auteur, mais une exploration de ce qu'est la vie humaine dans le piège qu'est devenu le monde.

Milan Kundera

Sans nous éloigner de la scène littéraire francophone, les héros romanesques du dernier quart de siècle évoluent, en général, dans des contextes sociologiques où les lecteurs peuvent plus ou moins se reconnaître. Ces personnages, qui se situent en haut ou en bas, à l'intérieur ou à l'extérieur de l'espace social considéré, interviennent dans un jeu qui reproduit la diversité et que Bajtine aurait nommée polyphonie. C'est grâce à l'échange de voix différentes, aux visions exotopiques, que la perception du nous peut aspirer à une relative totalité. ${ }^{9} \mathrm{De}$ la multiplicité de cultures découle celle des voix, celle des récits sur le plan littéraire.

De par son caractère mixte, d'autres diraient bâtard, le roman, et tout particulièrement le roman de la modernité, exprime et reproduit la nature hybride des cultures. Ce que l'on y trouve ce sont les espaces identitaires des marges, où les imaginaires renvoient davantage à l'expérience de l'exil, réel ou imaginaire, référés également à un espace présent ou absent. Il convient ici de rappeler que le thème de l'exil n'est pas aussi récent qu'on pourrait le croire. Au lieu de chanter avec Aimé Césaire le retour au pays natal, tous les migrants du monde entier disent plutôt leur départ de la terre d'origine. L'exil, comme expérience et comme image, a peut-être acquis de nouvelles modalités et d'autres formes d'expression, mais en fait il n'est

9 Totalité qui n'est jamais atteinte. Voir: Mijail M. BAJTıN, Yo también soy, fragmentos sobre el otro. 
qu'une variante, approfondie et diversifiée par la dynamique sociodémographique, de ce que, à d'autres époques, l'écrivain vivait comme une rupture ou comme la solitude face aux cadres établis et acceptés par les autres.

Il n'est donc pas étonnant que dans la production romanesque québécoise, mais aussi dans celle d'autres régions francophones, du dernier quart de siècle, l'un des thèmes récurrents soit celui du rapport à l'espace dans les grandes agglomérations urbaines définies par le plurilinguisme et par l'hétérogénéité culturelle. L'expérience de l'appartenance identitaire est particulièrement à réviser car la ou les langues en présence sur le même espace ne renvoient plus à un seul canon, et surtout pas à celui qui liait langue-culture-territoire. Dans ce cas, la contrainte ne joue plus dans le sens de l'uniformité, et l'aspiration à l'enracinement cesse parfois d'agir. "Peu à peu cependant, un processus insolite s'est mis en marche, discret et extrêmement efficace: accepter d'être étranger, exilé. Considérer toutes choses comme provisoires, être autre derrière mes apparences, me perdre dans ces rues propres et presque désertes, parmi des gens qui m'étaient inconnus", (p. 44) avoue le narrateur du Pavillon des miroirs.

Cette irruption décisive de l'hétérogène dans les lettres québécoises se produit quand la réalité sociopolitique crée quelques fisures dans un imaginaire que le discours postcolonialiste présentait encore comme homogène. Mais, pour les chercheurs intéressés par cette période, les nouvelles données imposées par l'hétérogénéité incontournable représentent plutôt une ouverture qu'une menace du fait que, en éliminant un centre fixe, la littérature peut mieux participer aux échanges qui se jouent dans les zones "périphériques" ou transfontalières des imaginaires convoqués.

\section{Conclusion}

Au terme de ces quelques réflexions, terme qui ne peut être que provisoire $v u$ le caractère foncièrement mouvant et pluriel de la réalité considérée, il conviendrait de saisir le sens dans lequel le discours littéraire qui se construit dans l'espace francophone, nous invite à nous engager pour analyser et comprendre ce qui se passe dans les sociétés contemporaines, notamment des pays à fort taux d'immigrants.

Les nouvelles générations -issues d'unions mixtes dans lesquelles convergent deux ou plusieurs héritages culturels et exposées, en dehors de l'enceinte familiale, au foisonnement d'autres manifestations culturelles-, ont des difficultés à revendiquer une appartenance identitaire bien définie. Les cadres de référence se sont diversifiés non seulement en raison du 
brassage de traditions différentes, mais aussi par la présence et l'influence des modèles culturels véhiculés par les mass média qui ne respectent aucune frontière. Il devient alors malaisé de dire où commence et où termine tel ou tel espace social, linguistique, culturel. Ces espaces pluriels, espaces de l'hétérogène, n'ont plus un centre unique.

Les différences culturelles définissant les individus ou les groupes face aux autres ne peuvent plus être abordées comme des totalités, mais plutôt au niveau du trait, du fragment. Accepter cette approche de l'hétérogénéité, non pas seulement comme extérieure mais aussi comme intérieure et intrinsèque au tissu social, ce sera accepter la dimension transculturelle de la société québécoise. Cela vaut également pour d'autres sociétés soumises à des processus sociodémographiques comparables et, par là même, définies par la diversité culturelle et linguistique. Il ne faut pas en effet perdre de vue - d'autres voix ont déjà exprimé ce voeu — que la projection du francais dépend du fait que chaque sous-région francophone puisse, dans un espace linguistique diversifié et en fonction de son génie et des besoins qui lui sont propres, créer et récréer son francais.

Dans son allocution "Egalités des langues, égalité des Etats", prononcée en 1998 à Genève, M.Mohammed Bedjaoui, juge à la Cour Internationale de Justice, déclarait: "qu'il n'y a aucune contradiction pour [le mouvement francophone] à développer autant qu'il le peut, ses solidarités internes, et en même temps à se rapprocher davantage de tous ceux qui tiennent au plurilinguisme, gage et expression de la diversité culturelle du monde". ${ }^{10}$

L'enjeu que la francophonie est tenue d'affronter est donc de faire en sorte que la langue francaise soit perçue et vécue comme un espace de "parcours transculturels", comme l'espace commun à toutes les expériences et où toutes les expressions sont concevables et admissibles. L'enjeu tient enfin aussi à l'adhésion aux politiques défendant le plurilinguisme qui visent à préserver et à faire partager les richesses de chaque culture dans les espaces définis par la diversité.

\section{Bibliographie}

AMSELLE, Jean-Loup, Lógiques métisses. Paris, Payot, 1990.

10 Le plurilinguisme dans les organisations internationales. Investir dans la diversité. Symposium de Genève, 5 et 6 novembre 1998, Organisation Internationale de la Francophonie, p. 26. 
BAJTIN, Mijail M., Yo también soy (fragmentos sobre el otro). Selec., trad. y pról. de Tatiana BuBNova. México, Taurus, 2000.

BeAuChemin, Yves, Juliette Pomerleau. Montreal, Québec/Amérique, 1994.

BISSOONDATH, Neil, Le Marché aux illusions, la méprise du multiculturalisme. Montreal, Boréal.Liber, 1995.

DEs RosıERs, Joël, Théories Caraïbes, Poétique du déracinement. Montreal, Tryptique, 1996.

GaGNON, Alain-G. et Alain Noël, L'Espace québécois. Montreal, Québec/ Amérique, 1995.

GaUvin, Lise, L'écrivain francophone à la croisée des langues. Paris, Karthala, 1997.

Houston, Nancy et Leilla Sebbar, Lettres parisiennes, Histoires d'exil. Paris, Editions J'ai lu, 1986.

KoKIS, Sergio, Le pavillon des miroirs. Montreal, X Y Z Editeur, 1995.

KUNDERA, Milan, L'art du roman. Paris, Gallimard, 1986.

LAFERRIÉre, Dany, Pays sans chapeau. Paris, Le Serpent à Plumes, 1999.

Le plurilinguisme dans les organisations internationales, Investir dans la diversité, Symposium de Genève, 5 et 6 novembre 1998, Organisation Internationale de la Francophonie.

MAALOUF, Amin, Identités meurtrières. Paris, Grasset, 1998.

MARCOTTE, Gilles et Pierre Nepveu, Montréal Imaginaire, Ville et littérature. Montreal, Fides, 1992.

NEPVEU, Pierre, L'Écologie du réel. Montreal, Boréal, 1999.

ROBIN, Régine, La Québécoite. Montreal, Québec/Amérique, 1983. 
SIMON, Sherry et al., Fictions de l'identitaire. Montreal, Etudes et documents, X Y Z, 1991.

\section{Articles}

FORTIN, Andrée, "Territoires culturels et déterritorialisation de la culture", La production culturelle en milieu minoritaire. Winnipeg, Presses Universitaires de Saint-Boniface, 1994, pp. 7-28.

LAFONTANT, Jean, "Interrogations d'un métèque sur la sybilline et dangeureuse notion d'identité collective", Sociologie et sociétés, vol. XXIV, n. 1. [Canada], printemps 1994, pp. 47-58.

LAFONTANT, Jean, "Langues, cultures et territoires, quels rapports?", Cahiers Franco-canadiens de l'ouest, vol. 7, n. 2. Manitoba, Collège de SaintBoniface, 1995, pp. 227-248.

LINTEAU, Paul André, "Les origines de la diversité culturelle au Québec", Textes du monde francophone, Dossier francophone. Coord. Christine Daumont-Spraag et Robert Cottrell. Montreal, Centre Educatif et culturel, 1987. 